Revue d'histoire de l'enfance « irrégulière »

Le Temps de l'histoire

$3 \mid 2000$

L'enfant de justice pendant la guerre et l'immédiat après-guerre

\title{
Wilfred Douglas Halls « Les jeunes et la politique de
} Vichy»

préface Jean-Pierre Rioux Paris, Syros, 1988, 502 p.

Jean-Jacques Yvorel

\section{OpenEdition \\ Journals}

\section{Édition électronique}

URL : http://journals.openedition.org/rhei/67

DOI : $10.4000 /$ rhei.67

ISBN : 978-2-7535-1640-3

ISSN : 1777-540X

Éditeur

Presses universitaires de Rennes

Édition imprimée

Date de publication : 15 novembre 2000

Pagination : $277-278$

ISSN : $1287-2431$

Référence électronique

Jean-Jacques Yvorel, « Wilfred Douglas Halls «Les jeunes et la politique de Vichy », Revue d'histoire de l'enfance « irrégulière » [En ligne], 3 | 2000, mis en ligne le , consulté le 04 décembre 2020. URL : http:// journals.openedition.org/rhei/67 ; DOI : https://doi.org/10.4000/rhei.67 


\author{
Wilfred Douglas Halls \\ Les jeunes et la politique de Vichy \\ préface Jean-Pierre Rioux \\ Paris, Syros, 1988, 502 p.
}

Publié en Anglais en 1981, traduit en 1988, le livre de Halls est la première synthèse sur Vichy et les jeunes. C'est toujours, sur le sujet, un ouvrage essentiel, rédigé à partir des fonds d'archives consultables.

La première partie, essentiellement politique, intitulée Les jeunes et leurs mentors, est divisée en cinq chapitres. Les deux premiers présentent les diverses tendances d'un "État français" très composite et rend compte des grands traits de l'évolution interne d'une politique pas vraiment cohérente. Il tracent parallèlement le portrait des hommes qui incarnèrent, en matière d'enseignement et de "jeunesse \& sport", la France de Vichy. Il conserve pour l'essentiel la distinction entre Old Guard et New Order introduite par Paxton dans son livre pionnier. Le troisième chapitre (L'Église et l'enseignement) analyse la politique de Vichy à l'égard de l'enseignement catholique. Le chapitre IV traite des enseignants et notamment des instituteurs. Il semble que le régime ne les aimait pas beaucoup et qu'ils n'aimèrent jamais beaucoup le régime. Le dernier chapitre de cette première partie se penche sur L'enrégimentation de la jeunesse et montre comment le projet d'une organisation unique pour la jeunesse, souhaité par les plus actifs partisans de la collaboration avec l'Allemagne, a échoué.'

La seconde partie traite du projet culturel et idéologique du gouvernement de Vichy. Les contradictions du régime ne sont pas moins aiguës en matière de réforme morale (chapitre VI) que lorsqu'il s'agit de déterminer la place du sport dans l'éducation générale (chapitre VII). Halls étudie ensuite Le révisionnisme culturel (chapitre VIII) et notamment les efforts de la Révolution nationale pour imposer une nouvelle lecture de l'histoire, qui gomme la Révolution française et ses conséquences. Enfin, contradiction fondamentale, le régime, même s'il compte quelques sympathies pour le régionalisme, invite la jeunesse à chanter la gloire d'une France

Jean-Jacques Yvorel / pp. 277 à 278 
une et indivisible, alors que les Allemands dépècent le territoire.

La troisième et dernière partie nous permet d'entrer plus avant dans le processus de mise au pas des jeunes français. En effet, Halls nous invite au sein même des organisations de jeunesse. Il consacre un court chapitre (15 pages) aux Compagnons de France, avant de s'étendre un peu plus sur les Chantiers de jeunesse. Les écoles de cadres, dont la célèbre école d'Uriage où s'expérimentèrent des méthodes pédagogiques actives qui inspirèrent parfois le champ de l'éducation spécialisée, ne sont pas oubliées (chapitre XII). Les organisations de jeunesse collaborationnistes et néo-nazies ne sont pas laissées dans l'ombre : nous les suivons jusqu'à leur ultime dérive et leur absortion dans la division SS Charlemagne. L'ouvrage est clos par un chapitre consacré au service du travail obligatoire en Allemagne. Le STO consomma le divorce entre un régime de plus plus fantoche et la jeunesse qu'un vieux maréchal avait rêvé de conquérir.

La synthèse de Halls n'est pas sans défaut. Ça et là nous trouvons quelques approximations comme, page 196, quand il attribue au régime de Vichy l'invention de la liberté surveillée et du tribunal pour enfants, institués en fait dès 1912 ! Surtout l'auteur, prisonnier de ses sources, privilégie une histoire institutionnelle, vue "d'en haut", qui laisse en définitive peu la parole aux jeunes. L'ouvrage n'en constitue pas moins une toile de fond indispensable pour qui veut travailler sur "l'enfant de justice" durant la guerre. 